\title{
ALEXANDER WEINSTEIN
}

1. Introduction. The subject of this paper is the theory of a special class of linear partial differential equations with variable coefficients which are rational functions of the independent variables. The type of differential equation considered here is of particular interest because it arises in a number of seemingly disconnected fields in pure and applied mathematics both classical and modern.

The simplest elliptic differential equation with constant coeffcients is the Laplace equation. Our class may be considered as the simplest among those with variable coefficients. Like Bessel's equation, which plays such a prominent role among ordinary differential equations with rational coefficients, our class is derived from the Laplace equation by introduction of a particular type of symmetry.

We shall deal mainly with elliptic equations, but in a natural way our subject will touch upon a class of hyperbolic equations of the Euler-Poisson type and on mixed equations of Tricomi's type.

Let us begin by considering a solution $f\left(x_{1}, x_{2}, \cdots, x_{n}\right)$ of the Laplace equation

$$
\frac{\partial^{2} f}{\partial x_{1}^{2}}+\frac{\partial^{2} f}{\partial x_{2}^{2}}+\cdots+\frac{\partial^{2} f}{\partial x_{n}^{2}}=0, \quad n>2 .
$$

We are interested only in axially-symmetric solutions of (1), namely, in those which depend on two variables $x=x_{1}$, and $y=\left(x_{2}^{2}+x_{3}^{2}+\cdots\right.$ $\left.x_{n}^{2}\right)^{1 / 2}$, the $x$-axis being the axis of symmetry. Under these restrictions $f$ becomes a function $\phi(x, y)$ of the two variables $x$ and $y$, and satisfies the equation

$$
\frac{\partial}{\partial x}\left(y^{n-2} \frac{\partial \phi}{\partial x}\right)+\frac{\partial}{\partial y}\left(y^{n-2} \frac{\partial \phi}{\partial y}\right)=0 .
$$

The case $n=3$ is classical and was investigated already in Laplace's time. Later, fundamental progress was made by Stokes in 1842 and above all by Beltrami [1] in his celebrated papers on symmetric potentials published about 1880 . While these investigations were concerned only with ordinary three-dimensional space, it was Arndt [2]

An address delivered before the New York meeting of the Society, February 23, 1952, by invitation of the Committee to Select Hour Speakers for Eastern Sectional Meetings; received by the editors May 19, 1952.

1 This work was performed under the sponsorship of the Office of Naval Research. 
who in 1915 first pointed out the importance of the case $n=5$ for the theory of torsion of shafts of revolution.

Let us consider in a more general way the equation

$$
\frac{\partial}{\partial x}\left(y^{p} \frac{\partial \phi}{\partial x}\right)+\frac{\partial}{\partial y}\left(y^{p} \frac{\partial \phi}{\partial y}\right)=0, \quad y \geqq 0,
$$

for all positive values of the parameter $p$. For all such values of $p$ we shall call $\phi$ an axially symmetric potential in a space of $n=p+2 \mathrm{di}$ mensions. For nonintegral values of $p$ this space is of course fictitious, but the corresponding equation (3) can be studied in the "meridian plane" $(x, y)$, where it still retains some of the properties of equation (2). The theory of our equations for general values of the parameter $p$ will be called generalized axially symmetric potential theory and will be abbreviated as GASPT. The integral values of $p$, especially $p=1$ $(n=3)$, will serve as a guide in the study of these generalized potentials. There is usually no great difficulty in rewriting for any positive value of $p$ the familiar and classical formulas of ordinary axially symmetric potential theory. For instance, the function $\left(x^{2}+y^{2}\right)^{-1 / 2}$ is replaced by $\left(x^{2}+y^{2}\right)^{-p / 2}$. The limiting case $p=0$ leads us back to the theory of harmonic functions in the $(x, y)$-plane. It will be seen that most of our results apply in this case provided the harmonic function is symmetric about the $x$-axis.

The introduction of nonintegral values of $p$ is not done for the sake of formal generalization. On the contrary, it will be shown that some interesting problems can be investigated by the method of GASPT.

2. The stream function and the generalized Stokes-Beltrami equations. Our differential equation (3) indicates the existence of a stream function $\psi(x, y)$ which is defined by the equations

$$
y^{p} \phi_{x}=\psi_{y} ; \quad y^{p} \phi_{y}=-\psi_{x} .
$$

We shall call (4) the generalized Stokes-Beltrami equations. Beltrami, who considered only the classical case $p=1$, was the first to point out the similarity to the Cauchy-Riemann equations. He showed by a process of repeated differentiation and integration that every potential or stream function generates a descending and ascending sequence of pairs of functions satisfying the same equations (4). For instance, in order to obtain Beltrami's first "derivative" $\left(\phi_{1}, \psi_{1}\right)$ of the pair $(\phi, \psi)$ we put

$$
\phi_{1}=\phi_{x} ; \quad \psi_{1}=\psi_{x} .
$$

On the other hand, the pair $\left(\phi_{*}, \psi_{*}\right)$, 


$$
\phi_{*}=\int\left(\phi d x-y^{-p} \psi d y\right) ; \quad \psi_{*}=\int\left(\psi d x+y^{p} \phi d y\right),
$$

will give us the "primitive function" of the pair $(\phi, \psi)$. In this way Beltrami founded a generalized theory of analytic functions which was later developed in a systematic way by Bers and Gelbart [3]. They considered equations of a more general type than (4) but restricted themselves exclusively to regular solutions, which do not concern us in this paper. Further details of this generalized theory of analytic functions can be found in the literature and will not be discussed further.

It follows from (4) that the stream function satisfies the equation

$$
\frac{\partial}{\partial x}\left(y^{-p} \frac{\partial \psi}{\partial x}\right)+\frac{\partial}{\partial y}\left(y^{-p} \frac{\partial \psi}{\partial y}\right)=0
$$

Obviously the equation for $\psi$ is obtained from the equation for $\phi$ by replacing $p$ by $-p$. Therefore, both $\phi$ and $\psi$ satisfy the equation

$$
\frac{\partial}{\partial x}\left(y^{k} \frac{\partial z}{\partial x}\right)+\frac{\partial}{\partial y}\left(y^{k} \frac{\partial z}{\partial y}\right)=0
$$

or

$$
z_{x x}+z_{y y}+k y^{-1} z_{y}=0,
$$

for $k=p$ and $k=-p$ respectively. It is, however, convenient in some applications to retain the notations $\phi$ and $\psi$ and to write $\phi\{p\}$ and $\psi\{p\}$ in order to emphasize the dependence upon the parameter $p$. In the same sense we shall use the notation $z\{k\}$. Since the line $y=0$ is a singular line for the coefficients of our equations, we shall usually restrict our considerations to the half-plane $y \geqq 0$.

3. The correspondence principle. The following simple but fundamental correspondence principle will be of great importance. It can be easily shown (see Weinstein [4]) that

$$
\psi\{p\}=C y^{p+1} \phi\{p+2\},
$$$$
p \geqq 0 \text {. }
$$

This result means that, given a $\psi\{p\}$, we can find a function $\phi\{p+2\}$ in two more dimensions, which is defined up to a constant factor $C$, and vice versa. In the notation of equation (9), equation (10) can be written as follows:

$$
z\{k\}=C y^{1-k}\{2-k\}, \quad-\infty<k<+\infty .
$$

In the following paragraphs we shall apply our principle to certain 
boundary value problems.

4. The hydrodynamical flow problem and the method of generalized electrostatics. The classical boundary value problem in axiallysymmetric hydrodynamics of incompressible perfect fluids is the determination of the flow about a body, the flow having a uniform velocity at infinity. It is an exterior Neumann problem for the potential $\Phi$. This potential can be written for any index $p$ in the form

$$
\Phi\{p\}=x-\phi\{p\},
$$

where the normal derivative $\partial \Phi / \partial \nu$ vanishes on the meridian profile of the body. At infinity we have for $\Phi$ a development of the type

$$
\Phi\{p\}=x+\frac{a x}{\left(x^{2}+y^{2}\right)^{(p+2) / 2}}+\cdots .
$$

The boundary condition $\partial \Phi / \partial \nu=0$ means that $\partial \phi / \partial \nu=\partial x / \partial \nu$ on the profile. The introduction of the stream function

$$
\Psi\{p\}=(p+1)^{-1} y^{p+1}-\psi\{p\}
$$

reduces our problem to an exterior Dirichlet problem. The stream function $\Psi\{p\}$ is single-valued and has at infinity the expansion

$$
\Psi\{p\}=(p+1)^{-1} y^{p+1}-\frac{a y^{p+1}}{\left(x^{2}+y^{2}\right)^{(p+2) / 2}}+\cdots .
$$

It vanishes on the $x$-axis and assumes a constant value $\Psi_{0}$ on the profile. If the profile and the $x$-axis form a single streamline, $\Psi_{0}$ will be zero. We shall discuss here only this last case in which the domain exterior to the profile is simply connected. The Dirichlet problem for $\Psi\{p\}$ is actually a Dirichlet problem for $\psi\{p\}$ which is of a rather complicated nature, since $\psi\{p\}$ must assume the value $(p+1)^{-1} y^{p+1}$ on the profile. For this reason the following new procedure (see Weinstein [5]) is more successful. We make use of (10) and replace $\psi\{p\}$ in (14) by $(p+1)^{-1} y^{p+1} \phi\{p+2\}$. In this way we obtain in place of (14) the following expression:

$$
\Psi\{p\}=(p+1)^{-1} y^{p+1}[1-\phi\{p+2\}] .
$$

The condition $\Psi\{p\}=0$ means that $\phi\{p+2\}=1$ on the profile. Moreover, it follows from (15) that $\phi\{p+2\}$ admits the expansion

$$
\phi\{p+2\}=\frac{(p+1) a}{\left(x^{2}+y^{2}\right)^{(p+2) / 2}}+\cdots
$$

at infinity. This means that formula (16) reduces the determination 
of $\Psi\{p\}$ to the determination of the electrostatic potential $\phi\{p+2\}$ of the body with the same meridian profile but in a space of two more dimensions. This simple but fundamental remark is based on the fact that we have the same factor $y^{p+1}$ in our correspondence principle (10) and in equation (14).

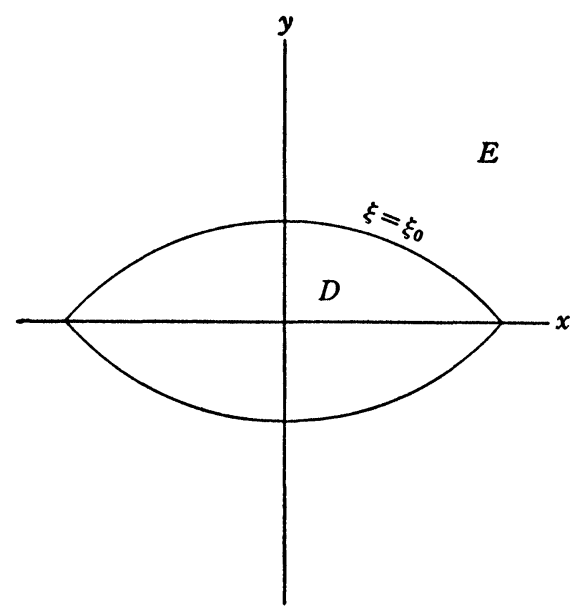

Fig. 1

A trivial example of our procedure is the determination of the flow about a sphere of unit radius which is usually obtained by putting a dipole at the origin. In contrast to this classical method we must merely find the electrostatic potential of a five-dimensional sphere, which is obviously $\left(x^{2}+y^{2}\right)^{-3 / 2}$. Thus we obtain immediately the familiar formula

$$
\Psi\{1\}=\frac{y^{2}}{2}\left[1-\left(x^{2}+y^{2}\right)^{-3 / 2}\right] .
$$

Naturally, the applications of our method of generalized electrostatics are not confined to such simple examples. In fact L. E. Payne [6] has recently obtained in this way the flow about a spindle (Fig. 1) which, despite its obvious importance, has not been treated previously.

In the case of the spindle we use dipolar coordinates defined by the equation

$$
x+i y=i c \cot (\xi+i \eta) / 2,
$$

where $c$ is an arbitrary positive constant. The range of coordinates is chosen as $-\infty<\eta<+\infty, 0<\xi \leqq \pi$. The profile of a spindle in the 
upper half-plane $y \geqq 0$ is a circular arc given by $\xi=\xi_{0}<\pi$. The region exterior to the profile is defined by $0<\xi<\xi_{0}$. The electrostatic potential of the three-dimensional spindle was first given by Mehler [7] in 1881. G. Szegö [8] in his address to the American Mathematical Society attributes it to $C$. Neumann. We need here, however, the electrostatic potential of a spindle in five-dimensional space. The generalization of Mehler's solution to any odd-dimensional space, a generalization which is far from trivial, is given by L. E. Payne [6]. By using equation (16), Payne obtains finally the following formula for the stream function for the flow about a spindle in a space of $n=p+2$ dimensions ( $n$ odd):

$$
\begin{array}{r}
\Psi\{p\}=\frac{(c \sin \xi)^{2(q+1)}}{2(q+1)(s-t)^{2(q+1)}}\left[1-\frac{2^{1 / 2} \Gamma(1 / 2)(s-t)^{q+3 / 2}}{\Gamma(q+3 / 2)}\right. \\
\left.\cdot \int_{0}^{\infty} \frac{K_{\alpha}^{(q+1)}\left(-t_{0}\right) K_{\alpha}^{(q+1)}(t) \cos \alpha \eta d \alpha}{K_{\alpha}^{(\alpha+1)}\left(t_{0}\right) \cosh \alpha \pi}\right] .
\end{array}
$$

In this formula

$$
s=\cosh \eta, \quad t=\cos \xi, \text { and } q=(p-1) / 2 .
$$

The symbol $K_{\alpha}^{a+1}(t)$ denotes the $(q+1)$-derivative of the conal function $K_{\alpha}(t)$ [9]. The classical case is of course given by $p=1$, and by inserting this value for $p$ in (20) we obtain the solution for a threedimensional spindle.

In order to evaluate the advantages of the method of generalized electrostatics, let us consider now the flow problem about a lens (Fig. 2). This flow was determined for the first time in 1947 by M. Shiffman and D. C. Spencer [10], who applied an ingenious and difficult procedure involving the method of images in a multi-sheeted Riemann-Sommerfeld space. In a much simpler way Payne obtains the solution [6] by a straightforward generalization to five dimensions of the electrostatic problem for the ordinary three-dimensional lens solved already in 1868 by Mehler [11]. Payne uses in place of (19) the peripolar transformation

$$
x+i y=-c \cot (\xi+i \eta) / 2, \quad c>0 .
$$

The profile of a lens is given by two intersecting circular arcs $\xi=\xi_{1}$ and $\xi=\xi_{2}$. We assume that $0<\xi_{1}<\xi_{2}<2 \pi$. The external region is given by the inequalities $\eta>0, \xi_{2}-2 \pi<\xi<\xi_{1}$. The stream function obtained by Payne in the most interesting case $n=3(p=1)$ is given by the formula 


$$
\begin{array}{r}
\Psi\{1\}=\frac{c \sinh ^{2} \eta}{2(\cosh \eta-\cos \xi)^{2}}\left[1+2^{3 / 2}(\cosh \eta-\cos \xi)^{3 / 2}\right. \\
\left.\cdot \int_{0}^{\infty} \frac{F(\alpha, \xi) K_{\alpha}^{(1)}(s)}{\cosh \alpha \pi} d \alpha\right],
\end{array}
$$

where

$$
F(\alpha, \xi)=\frac{\sinh \alpha\left(\xi_{1}-\xi\right) \cosh \alpha\left(\pi-\xi_{2}\right)+\cosh \alpha\left(\pi-\xi_{1}\right) \sinh \alpha\left(2 \pi-\xi_{2}+\xi\right)}{\sinh \alpha\left(2 \pi-\xi_{2}+\xi_{1}\right)}
$$

and $K_{\alpha}^{(1)}(s)$ denotes the first derivative of the Mehler function [9].

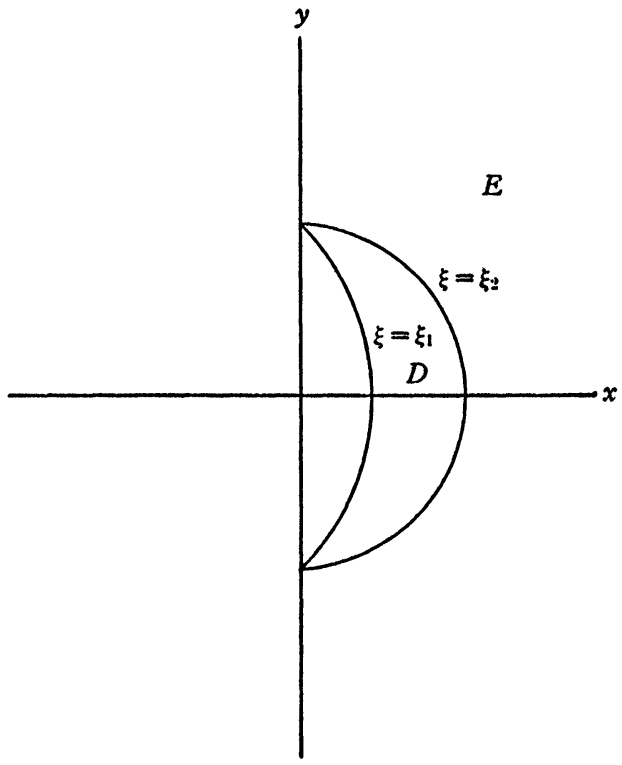

FIG. 2

There seems to be no elementary computational way of identifying Payne's results with those of Shiffman and Spencer. Of course, the identity of the two solutions is guaranteed by the uniqueness theorem. According to an oral communication from Professor Spencer, it would be difficult if not impossible to obtain the flow about a spindle by the method of images.

5. Torsion of shafts of revolution. Let us discuss here briefly another interesting application of the method of generalized electrostatics, namely, an application to the problem of the torsion of shafts, 
which has already been mentioned in the Introduction. This problem has been reduced by Arndt to the determination of two functions $\Phi$ and $\Psi$ defined in the meridian plane and satisfying equations (4) for $p=3$. The profile of the shaft is defined by lines $\Psi=\Psi\{3\}=$ constant.

$\Psi\{3\}=$ const.

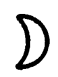

FIG. 3

Therefore, the problem of torsion is essentially equivalent to the flow problem in a five-dimensional space. For instance, the flow about a closed profile on which $\Psi\{3\}=0$ (see Fig. 3) can be interpreted as the solution of a torsion problem for a shaft with a cavity, the outer boundary of the shaft being given by lines $\Psi\{3\}=$ constant. For moderately large values of this constant the outer boundary of the profile deviates only slightly from a parallel to the $x$-axis. According to the correspondence principle (10) the five-dimensional flow problem may be interpreted as an electrostatics problem in a space of seven dimensions. In this way several problems in the torsion of shafts in ordinary three-dimensional space have recently been solved by an application of the theory of electrostatics in a seven-dimensional space (see Weinstein [12]).

6. Capacity, virtual mass, and generalized symmetrization. The most important functional in electrostatics is the capacity of a conductor. For bodies of revolution in a space of any number of dimensions the capacity can be defined as a Dirichlet integral in the $(x, y)$ plane taken over the region $E$ exterior to the profile of the body. As always, we restrict ourselves to non-negative values of $y$.

In the following we shall of ten use notations like $\phi[n]$ in place of $\phi\{p\}$ to indicate the number of dimensions. In this notation the capacity $C[n]$ corresponding to a body $B[n]$ with a meridian section $D$ is given by the following formula, which generalizes the classical case $n=3$ :

$$
C[n]=\omega_{n-1} \omega_{n}^{-1} \iint_{E} y^{n-2}(\operatorname{grad} \phi[n])^{2} d x d y .
$$

As in previous paragraphs, we take the same section $D$ for all values of $n$. The $\omega$ 's in (23) denote areas of unit hyperspheres. The function 
$\phi[n]$ represents the electrostatic potential already introduced in section 4.

For the hydrodynamical problem the most important quantity is the virtual mass $M[n]$ of $B[n]$ for a flow in the $x$-direction. Putting the density and the velocity at infinity equal to unity, we have

$$
M[n]=\omega_{n-1} \iint_{E} y^{n-2}(\operatorname{grad} \phi[n])^{2} d x d y .
$$

Let us note that the potential $\phi[n]$ in (24) is not the electrostatic potential but the velocity potential introduced by equation (12). Using the associate function $\psi[n]$, we may replace formula (24) by

$$
M[n]=\omega_{n-1} \iint_{E} y^{-(n-2)}(\operatorname{grad} \psi[n])^{2} d x d y .
$$

By the correspondence principle we can introduce in place of $\psi[n]$ the electrostatic potential $\phi[n+2]$. An integration by parts leads then to the following fundamental formula, given by Payne and Weinstein [13],

$$
M[n]+V[n]=\pi^{n / 2}\left[(n-1) \Gamma\left(\frac{n}{2}+1\right)\right]^{-1} C[n+2],
$$

where

$$
V[n]=\omega_{n-1} \iint_{D} y^{n-2} d x d y .
$$

Obviously $V[n]$ is the $n$-dimensional volume of the body $B[n]$. For $n=3$ formula (26) was given already by G. I. Taylor [14] and mentioned by Schiffer and Szegö [15]. In these papers the right-hand side of (26) was called a dipole coefficient. However, it is advantageous to interpret the dipole coefficient as a capacity in $(n+2)$ dimensions because numerous known results for the capacity of ordinary three-dimensional bodies of revolution remain valid for any value of $n$. Thus, the fundamental formula (26) may be used for the study of the virtual mass. In this connection let us now say a few words about generalized symmetrization.

The influence of symmetrization on the capacity has been recently investigated by numerous authors, the foremost of whom are Pólya and Szegö [16]. Some particular results for the virtual mass have been obtained by Garabedian and Spencer [17]. It is interesting to note that the method of generalized electrostatics not only gives simpler 
proofs of several known results but also leads to a natural extension of the concept of symmetrization.

Let us consider an ordinary body of revolution $B[3]$ in a threedimensional space. A line $x=$ constant, $y \geqq 0$ will intersect the profile $L$ of $B$ in $m$ points, $y_{1}(x)>y_{2}(x)>\cdots>y_{m}(x)>0$. It is obvious that the number of intersections $m$ depends upon $x$. (See, for example, Fig. 2.) This body can be symmetrized with respect to its axis of symmetry in a number of ways. One of these is the Schwarz symmetrization, which preserves the volume of $B[3]$. Another is the Steiner symmetrization of the meridian section of $B[3]$, which preserves the area of the section but in general decreases the volume. Under Schwarz symmetrization the profile $L$ is replaced by a new profile $L_{2}$ given by the equation $y^{2}(x)=y_{1}^{2}(x)-y_{2}^{2}(x)+\cdots$. In the case of Steiner symmetrization the profile $L$ is replaced by another curve $L_{1}$ defined by the equation $y(x)=y_{1}(x)-y_{2}(x)+\cdots$. This leads to the following generalization. Let us define a profile $L_{q}$ by the equation

$$
y^{q}(x)=\sum_{k=1}^{m}(-1)^{k-1} y_{k}^{q}(x)
$$

where $q$ is any positive integral or nonintegral number. We say that the profile $L_{q}$ given by the function $y(x)$ has been obtained from $L$ by a generalized symmetrization $S_{q}$. From now on let us assume, as in section 4 , that for any $n \geqq 3$ all profiles are boundaries of bodies of revolutions in an $n$-dimensional space. We can then prove the following three theorems (see Payne and Weinstein [13]):

I. $V[n]$ does not increase under $S_{q}$ for $0<q \leqq n-1$ and does not decrease under $S_{q}$ for $q \geqq n-1$. In particular, $V[n]$ remains invariant under $S_{n-1}$.

II. $C[n]$ does not increase under $S_{q}$ for $0<q \leqq n-1$.

III. $M[n]$ does not increase under $S_{q}$ for $n-1 \leqq q \leqq n+1$. Let us observe that, by (26), Theorem III follows immediately from Theorems I and II.

The main tools for the proofs of these theorems are the inequalities

$$
\left[\sum_{k=1}^{m}(-1)^{k-1} y_{k}^{q+s}\right]^{1 /(q+s)} \leqq\left[\sum_{k=1}^{m} y_{k}^{q}\right]^{1 / q}
$$

and

$$
\left[\sum_{k=1}^{m}(-1)^{k-1} y_{k}^{q}\right]^{1 / q} \leqq\left[\sum_{k=1}^{m}(-1)^{k-1} y_{k}^{q+s}\right]^{1 /(q+s)},
$$


which are valid for positive $q$ and $s$ when $y_{1}>y_{2}>\cdots>y_{m}>0$. The first of these inequalities is classical. The proof of the second inequality is due to H. F. Weinberger and can be found in [13].

Theorem I follows immediately from (29) and (30). As to Theorem II concerning the capacity, we proceed following the pattern given in [16]. We introduce a function $z(x, y)$ which is constant over the domain $D$ and vanishes on the boundary of a large half-circle $A$. We find, using (29), that the integral generalizing the surface area

$$
I=\iint_{A} y^{n-2}\left[1+z_{x}^{2}+z_{y}^{2}\right]^{1 / 2} d x d y
$$

does not increase under a symmetrization $S_{n-1}$. Let us now replace $z(x, y)$ by $\epsilon \chi(x, y)$ where $\epsilon$ is a small positive number tending to zero. Then we find, by using Theorem I, that the integral

$$
\iint_{A} y^{n-2}\left(\chi_{x}^{2}+\chi_{y}^{2}\right) d x d y
$$

does not increase under $S_{n-1}$. By letting the radius of $A$ tend to infinity, we find that we can replace $\chi$ in (32) by the electrostatic potential $\phi[n]$ and the domain of integration $A$ by the exterior $E$ of $D$. In this way it is shown that $C[n]$ does not increase under $S_{n-1}$. Let us now consider the influence of the symmetrization $S_{q}$ for $0<q \leqq n-1$. According to the inequality (30) no point of the profile $L_{q}$ lies outside of $L_{n-1}$. The well known monotone property of the capacity remains valid in any number of dimensions. We see, therefore, that under $S_{q}$ the capacity is a nondecreasing function of $q$. This remark concludes the proof of Theorem II.

7. Existence and uniqueness theorems for data on the singular line. In the previous paragraphs we have been chiefly concerned with certain classical boundary value problems in GASPT. Let us now turn to the general theory of our associated equations (3) and (7), which we may replace by the single equation (9) considered for all real values of $k$. For positive values of $y$ this equation is elliptic with regular analytic coefficients. Therefore, as long as $y$ is positive, the general theory of such equations applies. However, the $x$-axis is a singular line for the coefficients of (9). In a certain sense there is an analogy between our equation and ordinary differential equations with a singular end point. As is well known, equations such as Bessel's equation admit some solutions which remain regular at this point. Similarly we have some functions $z$ which remain regular on the $x$-axis, for example, $z=x$. For such solutions the following unique- 
ness and existence theorems are of importance.

If $k \neq 0,-1,-2, \cdots$, then there is one and only one function $z\{k\}$ which is regular analytic in a region containing a segment of the $x$-axis in its interior, and which takes prescribed analytic values $g(x)$ on this segment. This function $z$ is necessarily an even function of $y$. If $k=0,-2,-4, \cdots$, the solution $z\{k\}$ always exists for arbitrary analytic values on the $x$-axis but is determined only up to an additive function of the type $y^{1-k} z\{2-k\}$, where the values of $z\{2-k\}$ on the $x$-axis are arbitrary. If, however, $k=-1,-3,-5, \cdots$, then $z\{k\}$ exists only if the analytic values on the $x$-axis are given as an arbitrary polynomial in $x$ of degree not exceeding $-k$. Again, in this case, the function $z\{k\}$ is determined only up to an additive function $y^{1-k} z\{2-k\}$. This indetermination is to be expected in view of the correspondence principle.

In this generality the existence and uniqueness theorems were given by Hyman [18], who used a power series expansion of $z\{k\}$. For positive $k$ a uniqueness theorem had already been given by Weinstein [4]. It should be noted also that for $k>0, z\{k\}$ can be explicitly given as a Laplace integral,

$$
z\{k\}=C_{k} \int_{0}^{\pi} g(x+i y \cos \alpha) \sin ^{k-1} \alpha d \alpha
$$

where $C_{k}^{-1}=2^{k-1} \Gamma^{2}(k / 2) \Gamma^{-1}(k)$. A generalization of (33) for $k \leqq 0$ will be given in a forthcoming paper by Diaz and Weinstein.

8. The fundamental solution. The uniqueness theorem of section 7 may be used as an identification principle permitting us to identify a complicated solution by its values on the $x$-axis. The simplest example is the Lipschitz integral

$$
\phi_{0}\{p\}=\frac{2 y^{-q} \Gamma(q+1)}{\Gamma(2 q+1)} \int_{0}^{\infty} t^{q} e^{-|x| t} J_{q}(y t) d t=\left(x^{2}+y^{2}\right)^{-p / 2} .
$$

This integral may be identified by its values on the $x$-axis, namely, $|x|^{-p}$. In (34) we have $p>0$ and $q=(p-1) / 2$. This solution has a singularity on the $x$-axis. The first step of the general theory, already clearly recognized by Beltrami for $p=1$, is to find a fundamental solution with a logarithmic singularity at an arbitrary point above the $x$-axis, say at $x=0, y=b>0$. A fundamental solution has the form

$$
\phi\{p\}=u(x, y) \log \left[x^{2}+(y-b)^{2}\right]+v(x, y),
$$

where $u$ and $v$ are regular analytic at the singular point. In the classical case $p=1(n=3)$ Beltrami points out that such a solution would 
correspond to the potential of a uniform ring with the point $b$ as the trace in the meridian half-plane. By our identification principle this solution can be easily constructed for $p=1$ due to the fact that it takes on the $x$-axis the value $\left(x^{2}+b^{2}\right)^{-1 / 2}$. Beltrami's result suggests that for $p>0$ the fundamental solution defined in the entire halfplane $y \geqq 0$ will be the solution taking the value $\left(x^{2}+b^{2}\right)^{-p / 2}$ on the $x$-axis. There is a bewildering variety of forms of such a solution, all of which coincide by the identification principle. The formula [4]

$$
\phi\{p\}=\pi C_{p}(b y)^{-q} \int_{0}^{\infty} e^{-|x| t} J_{q}(y t) J_{q}(b t) d t, \quad q=\frac{p-1}{2}
$$

is a direct generalization of Beltrami's formula for $p=1$. The constant $C_{p}$ has been introduced in (33). Another expression (see Weinstein [4]), which shows more easily that $\phi\{p\}$ is actually of the form (35), is given by

$$
\phi\{p\}=C_{p} \int_{0}^{\pi}\left(x^{2}+y^{2}+b^{2}-2 b y \cos \alpha\right)^{-p / 2} \sin ^{p-1} \alpha d \alpha .
$$

9. The stream function and the integrals of Weber-Shafheitlin. It may be surmised that Beltrami chose the expression (36) for the fundamental solution because it was then easy to compute the associate function $\psi$. As $|x|$ is equal to $x$ or $-x$ according to whether $x$ is positive or negative, we obtain, generalizing the computations of Beltrami, the following expressions for $\psi$ :

$$
\begin{array}{ll}
\psi^{+}=-\pi C_{p} b^{-q} y^{q+1} \int_{0}^{\infty} e^{-x t} J_{q+1}(y t) J_{q}(b t) d t, & x \geqq 0, \\
\psi^{-}=\pi C_{p} b^{-q} y^{q+1} \int_{0}^{\infty} e^{x t} J_{q+1}(y t) J_{q}(b t) d t=-\psi^{+}, & x \leqq 0 .
\end{array}
$$

At this point Beltrami, who considers only the case $q=0$, makes a fundamental mistake in not realizing that the associate function $\psi$ is a many-valued function and that $\psi^{+}$and $\psi^{-}$represent two of its branches which do not coincide on a part of the $y$-axis. Beltrami's error has often been repeated. We shall show that this error is more than a historic curiosity as the investigation of the situation leads to an interesting extension of the theory of discontinuous integrals. As $\partial \phi / \partial x=0$ on the $y$-axis, we see that $\psi^{+}$takes constant values on the interval $0 \leqq y<b$ and the interval $b<y<\infty$. The first of the constants is zero as $\psi^{+}$is zero at the origin. Let us now apply the relationship 


$$
\int y^{p} \frac{\partial \phi}{\partial \nu} d s=\int \frac{\partial \psi^{+}}{\partial s} d s
$$

to an infinitesmal arc of a circle with center at $b$. The computation of

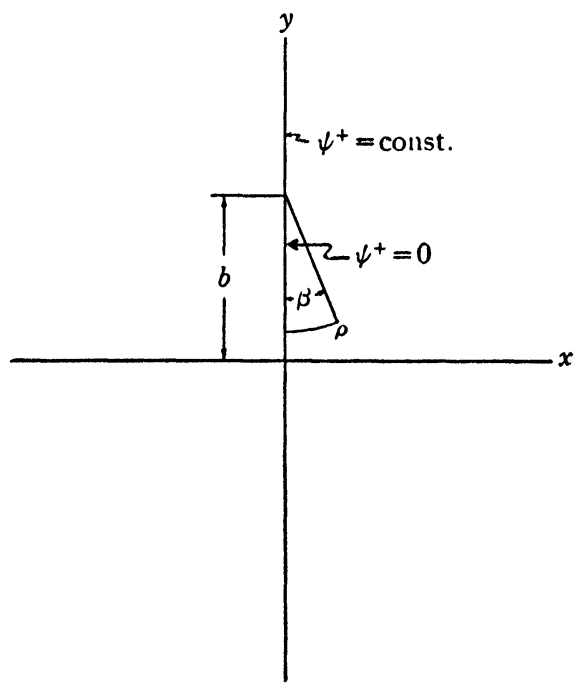

FIG. 4

the residue of the integral on the left shows that $\psi^{+}$is locally proportional to the angle $\beta$ (Fig. 4). This leads to the fundamental formula (see Weinstein [4])

$$
\lim y^{q+1} \int_{0}^{\infty} e^{-x i} J_{q+1}(y t) J_{q}(b t) d t=\frac{\beta}{\pi} b^{q}
$$

where $P \rightarrow(0, b)$ along a ray. Let us note that, for integral values of $p$, Van Tuyl [19] and also Sadowsky and Steinberg [20] expressed the many-valued function $\psi$, defined by (38) and (39), in terms of elliptic integrals. The formula (41) contains as a special case the results of Weber and Schafheitlin. In fact, by taking $\beta=\pi$, we obtain not only the limit but the constant value of $\psi^{+}$on the interval $b<y<\infty$, namely $-\pi C_{p}$. Choosing $\beta=\pi / 2$, we obtain $\psi^{+}=-(\pi / 2) C_{p}$ at the branch point. These results are obviously equivalent to the classical Weber-Shafheitlein formula

$$
\int_{0}^{\infty} J_{q+1}(y t) J_{q}(b t) d t= \begin{cases}0, & y<b \\ (2 b)^{-1}, & y=b \\ b^{q} y^{-(q+1)}, & y>b\end{cases}
$$


Thus, our theory establishes a connection between discontinuous integrals and many-valued functions.

10. The Green's function for a half-plane and Poisson's integral. Let us use again the notation $z\{k\}$ instead of $\phi\{p\}$ and $\psi\{p\}$. In section 8 we gave several expressions which represent a fundamental solution $z\{k\}, k>0$, in the half-plane $y \geqq 0$. The equation (11), $z\{k\}=C y^{1-k} z\{2-k\}$, permits us to find new fundamental solutions in the large which are of particular interest in the interval $-\infty<k<1$. They are obtained by taking for $z\{2-k\}$ the fundamental solutions corresponding, in the presentation notation, to (36) or (37). As these new fundamental solutions vanish on the $x$-axis, we have thus established an explicit formula for the Green's function belonging to the half-plane $y \geqq 0$ for the equation $z_{x x}+z_{y y}+(k / y) z_{y}=0$, for $-\infty<k$ $<1$. Corresponding to this Green's function one has a Poisson's integral formula

$$
z\{k\}=A y^{1-k} \int_{-\infty}^{\infty} \frac{f(\xi) d \xi}{\left[(x-\xi)^{2}+y^{2}\right]^{(2-k) / 2}},
$$

which solves the Dirichlet problem for the half-plane $y \geqq 0$, for given bounded continuous boundary values $f(\xi)$. The numerical constant $A$ is determined by putting $f(\xi) \equiv 1$. As it will be shown in a forthcoming paper, Green's function does not exist for $k \geqq 1$.

11. Tricomi's equation. One of the most interesting applications of GASPT is a new approach, given by Weinstein [22], to the theory of Tricomi's equation

$$
\eta z_{\xi \xi}+z_{\eta \eta}=0, \quad z=z(\xi, \eta) .
$$

This is the simplest equation of mixed type. It is elliptic in the upper half-plane $\eta>0$ and hyperbolic in the lower half-plane $\eta<0$.

In the hyperbolic half-plane $\eta<0$ let us put

$$
x=\xi, \quad t=2(-\eta)^{3 / 2} / 3 .
$$

In this way we get as a normal form for Tricomi's equation an Euler-Poisson equation

$$
z_{x x}-z_{t t}-\frac{1}{3 t} z_{t}=0,
$$

which belongs to the type of Euler's equation treated by Riemann in his celebrated memoir on gas dynamics. Equation (46) was used by Tricomi and his successors as a main road of access to the general 
study of the mixed equation (44). More recently, the following new approach has been proposed $[21 ; 22]$. Let us first consider the elliptic half-plane $\eta>0$. Tricomi's equation can be reduced by the transformation

$$
x=\xi, \quad y=2 \eta^{3 / 2} / 3, \quad \eta>0,
$$

to the equation

$$
z_{x x}+z_{y y}+\frac{1}{3 y} z_{y}=0
$$

which is an axially symmetric potential in $2 \frac{1}{3}$ dimensions. Therefore, the results of GASPT can be applied immediately.

Before proceeding, let us make the following simple remark. To any solution of the equation

$$
z_{x x}+z_{y y}+\frac{k}{y} z_{y}=0, \quad k \gtrless 0,
$$

there corresponds a solution of the equation

$$
z_{x x}-z_{t t}-\frac{k}{t} z_{t}=0, \quad k \gtrless 0,
$$

which is obtained from (48) by putting $y=i t$. For instance, to the Laplace integral (33) corresponds the following solution of Euler's equation:

$$
z\{k\}=C_{k} \int_{0}^{\pi} g(x+t \cos \alpha) \sin ^{k-1} \alpha d \alpha, \quad, k>0, z_{t}(x, 0)=0,
$$

which is a celebrated formula given by Poisson.

For certain rational values of the parameter $k$ the equations (49) and (50) are connected by an equation of Tricomi's type (44) in which the factor $\eta$ is replaced by $\eta^{2 m+1}, m=0, \pm 1, \pm 2, \cdots$.

Let us now return to Tricomi's equation. A question of paramount interest, widely discussed in recent years, is the determination of a fundamental integral which is defined in the entire plane and has a logarithmic singularity in the elliptic half-plane.

The simplest way to obtain such an integral is to take the fundamental solution (37) corresponding to a ring and rewrite it in the $\xi, \eta$ coordinates for $\eta>0$. This solution takes the value

$$
z(\xi, 0)=\left(\xi^{2}+(4 / 9) \beta^{3}\right)^{-1 / 6}, \quad(4 / 9) \beta^{8}=b^{2},
$$

on the $\xi$-axis while $z_{\eta}=0$. Then, using Poisson's formula (51) for 
Euler's equation, we obtain an analytic continuation for all $\eta \leqq 0$ given by

(53) $z(\xi, \eta)=C_{1 / 3} \int_{0}^{\pi}\left[\left(\xi+(2 / 3)(-\eta)^{3 / 2} \cos \alpha\right)^{2}+(4 / 9) \beta^{3}\right]^{-1 / 6} \sin ^{-2 / 3} \alpha d \alpha$.

This procedure indicates the fruitful correspondence between GASPT, the Euler-Poisson-Riemann equation, and Tricomi's equation.

12. Concluding remarks. The review of GASPT given in the preceding paragraphs is by no means complete. We have omitted numerous applications such as transonic flow (Weinstein [22]), the method of sources and sinks (Weinstein $[23 ; 24]$ ), the theory of dislocations (Weinstein [12]), and turbulent diffusion [25]. Neither did we mention the extension of the Riemann's integration method as given by Martin [26] and later by Diaz and Martin [27] in which a pair of associate Euler-Poisson equations plays an important part in the Cauchy problem for the wave equation.

Finally, there is an important extension of GASPT to equations for functions $z\left(x_{1}, \cdots, x_{m}\right)$ of $m>2$ variables. The equation (9) is here replaced by

$$
\frac{\partial^{2} z}{\partial x_{1}^{2}}+\cdots+\frac{\partial^{2} z}{\partial x_{m-1}^{2}}+\frac{\partial^{2} z}{\partial x_{m}^{2}}+\frac{k}{x_{m}} \frac{\partial z}{\partial x_{m}}=0
$$

which is the Beltrami-Laplace equation on a Riemannian manifold defined by the metric

$$
d s^{2}=\frac{d x_{1}^{2}+\cdots+d x_{m}^{2}}{\left(x_{m}\right)^{2 k /(2-m)}} .
$$

This metric is of particular interest, since a special case $(k=2-m)$ of (55) is the $d s^{2}$ of a Poincaré manifold of constant negative curvature -1 . We note finally that there is a formal correspondence between (54) and the Euler-Poisson-Darboux equation in several variables which plays such an important role in the integration of the wave equation. These questions, however, would lead us into some problems concerning hyperbolic equations, which lie beyond the scope of the present address [28].

Added in proof (January 27, 1953). In a paper presented to the American Mathematical Society, A. Huber extended considerably the results of $\$ 7$ by proving the following uniqueness theorem: If $z\{k\}$ is a solution of (54) assuming the boundary value 0 on an open sub- 
set $S$ of the hyperplane $x_{m}=0$, then, for $k \geqq 1, z\{k\} \equiv 0$, and for $k<1, z\{k\} \equiv x_{m}^{1-k} z\{2-k\}$, where $z\{2-k\}$ is analytic also on $S$. A fundamental solution in the large for (54) has been recently given by Diaz and Weinstein in a paper to be published in the Anniversary Volume for $\mathrm{R}$. von Mises.

\section{BIBLIOGRAPHY}

1. E. Beltrami, Opere Matematiche, vol. 3, Milano, Hoepli, 1911, pp. 349-382.

2. W. Arndt, Die Torsion von Wellen mit achsensymmetrischen Bohrungen und Hohlräumen, Dissertation, Göttingen, 1916.

3. L. Bers and A. Gelbart, On a class of differential equations in mechanics of continua, Quarterly of Applied Mathematics vol. 1 (1943) pp. 168-188.

4. A. Weinstein, Discontinuous integrals and generalized potential theory, Trans. Amer. Math. Soc. vol. 63 (1948) pp. 342-354.

5. - The method of singularities in the physical and in the hodograph plane, Fourth Symposium of Applied Mathematics (in print).

6. L. E. Payne, On axially symmetric flow and the method of generalized electrostatics, Quarterly of Applied Mathematics vol. 10 (1952) pp. 197-204.

7. F. G. Mehler, Ueber eine mit dem Kugel und Cylinderfunctionen verwandte Function und ihre Anwendung in der Theorie der Elektricitätvertheilung, Math. Ann. vol. 18 (1881) pp. 161-194.

8. G. Szegö, On the capacity of a condenser, Bull. Amer. Math. Soc. vol. 51 (1945) pp. 325-350. 1931.

9. E. Hobson, Spherical and ellipsoidal harmonics, Cambridge University Press,

10. M. Shiffman and D. C. Spencer, The flow of an ideal incompressible fluid about a lens, Quarterly of Applied Mathematics vol. 5 (1947) pp. 270-288.

11. F. G. Mehler, Ueber die Vertheilung der statischen Elektricität in einem von zwei Kugelkalotten begrenzten Körper, J. Reine Angew. Math. vol. 68 (1868) pp. 134-150.

12. A. Weinstein, On cracks and dislocations in shafts under torsion, Quarterly of Applied Mathematics vol. 10 (1952) pp. 77-81.

13. L. E. Payne and A. Weinstein, Capacity, virtual mass and generalized symmetrization, Pacific Journal of Mathematics vol. 2 (1952) pp. 633-641.

14. G. I. Taylor, The energy of a body moving in an infinite fluid with an application to airships, Proc. Roy. Soc. London ser. A vol. 120 (1928) pp. 21-33.

15. M. Schiffer and G. Szegö, Virtual mass and polarization, Trans. Amer. Math. Soc. vol. 67 (1949) pp. 130-205.

16. G. Polya and G. Szegö, Isoperimetric inequalities in mathematical physics, Annals of Mathematics Studies, no. 27, Princeton University Press, 1951.

17. P. R. Garabedian and D. C. Spencer, Extremal methods in cavitational flow, Technical Report No. 3, Appl. Math. and Stat. Lab., Stanford University, 1951.

18. M. Hyman, Abstracts (Preliminary Reports), Bull. Amer. Math. Soc. vol. 54 (1948) p. 1065 ; vol. 55 (1949) pp. 284-285.

19. A. Van Tuyl, On the axially symmetric flow around a new family of halfbodies, Quarterly of Applied Mathematics vol. 7 (1950) pp. 399-409.

20. M. A. Sadowsky and E. Sternberg, Elliptic integral representation of axially symmetric flows, Quarterly of Applied Mathematics vol. 8 (1950) pp. 113-126.

21. A. Weinstein, On Tricomi's equation and generalized axially symmetric potential theory, Bull. Acad. Royale de Belgique vol. 37 (1951) pp. 348-358. 
22. - Transonic flow and generalized potential theory, Proc. Aeroball. Res. Symposia, Naval Ordnance Laboratory (1949) pp. 73-82.

23. - On axially symmetric flows, Quarterly of Applied Mathematics vol. 5 (1948) pp. 429-444.

24. - On the torsion of shafts of revolution, Proc. 7th Internat. Cong. Appl. Mech., vol. 1, 1948, pp. 108-119.

25. D. R. Davies and T. S. Walters, The effect of finite width of area on the rate of evaporation into a turbulent atmosphere, Quart. J. Mech. and Appl. Math. vol. 4 (1951) pp. 466-480.

26. M. H. Martin, Riemann's method and the problem of Cauchy, Bull. Amer. Math. Soc. vol. 57 (1951) pp. 238-249.

27. J. B. Diaz and M. H. Martin, Riemann's method and the problem of Cauchy, II. The wave equation, Proc. Amer. Math. Soc. vol. 3 (1952) pp. 476-483.

28. A. Weinstein, Sur le problème de Cauchy pour l'equation de Poisson et l'equation des ondes, C. R. Acad. Sci. Paris vol. 234 (1952) pp. 2584-2585.

UNIVERSITY OF MARYLAND 